\title{
Shifting emphasis from pharmacogenomics to theragnostics
}

\author{
Vural Ozdemir ${ }^{1,2}$, Bryn Williams-Jones ${ }^{3}$, Stephen J Glatt ${ }^{4}$, Ming T Tsuang ${ }^{4}$, James B Lohr ${ }^{2,4,5}$ \& Christopher Reist ${ }^{1,2}$ \\ What will be the role of theragnostic patents in upstream and downstream biomarker research?
}

Phar harmacogenomics aims to identify the genetic basis of variability in drug efficacy and safety, and ultimately develop diagnostics that can individualize pharmacotherapy. Theragnostics, a term denoting the fusion of therapeutics and diagnostics, is receiving increasing attention as pharmacogenomics moves to applications at point of patient care. In contrast to pharmacogenomics, theragnostic tests focus not on a singular marker set, such as genetic polymorphisms, but rather on the integration of information from a diverse set of biomarkers (e.g., genomic, proteomic, metabolomic).

Although it remains to be seen whether theragnostics reflects a form of hyperbole in biomarker research, it is grounded in both established (e.g., genomics) and exploratory (e.g., metabolomics) technologies that can offer, respectively, mechanistic and heuristic insights for therapeutics (Fig. 1). Recent social science analyses suggest that in some cases, bio-

\footnotetext{
${ }^{1}$ Biomarker and Clinical Pharmacology Unit, VA Long Beach Healthcare System, Southern California Institute for Research and Education and Department of Psychiatry and Human Behavior, University of California, Irvine, CA, USA. ${ }^{2}$ Mental Illness Research, Education, and Clinical Center, VISN 22, Long Beach, San Diego and Los Angeles. ${ }^{3}$ Groupe de recherche en bioéthique \& Département de médicine sociale et préventive, Université de Montréal, Montréal, Québec, Canada. ${ }^{4}$ Center for Behavioral Genomics, Department of Psychiatry, University of California, San Diego, CA, USA. ${ }^{5}$ Psychopharmacology Research Initiatives Center of Excellence, University of California, San Diego, CA, USA. e-mail: creist@uci.edu,vural.ozdemir@med. va.gov,bryn.williams-jones@umontreal.ca
}

hype can be an integral component or driver for the establishment of biotechnologies, particularly in the early stages of development of a new concept or idea ${ }^{1-5}$. The synthesis of both types of technologies (established and exploratory) will likely have a differential impact on the regulation and economic promise of pharmaceuticals developed under the overarching theme of theragnostics. Moreover, we suggest that advances in this field may shape, in potentially unexpected ways, the pursuit of theragnostic patents depending on whether the research is conducted in an upstream drug discovery-oriented context or towards downstream point-of-care applications $s^{6,7}$.

As biomarker applications move towards point-of-care to individualize drug therapy, a number of qualitatively different concerns arise relating to gene patents and ethical and therapeutic policy aspects of theragnostic testing $^{4,8,9}$. For example, a fear is that theragnostic tests could be adopted without regard for the particular research context in which they are being applied, or be understood as a homogeneous category, a ubiquitous set of biotechnologies with similar implications for therapeutic policy ${ }^{6,9-11}$. In effect, this may result in a predicament where the patents on biomarkers are conceptualized in a one-size-fits-all manner (as in drug prescriptions) thereby precluding the equitable implementation of emerging biomarker technologies and informed critique of their societal implications. Additionally, the effects of theragnostic patents on 'knowledge commons' - that is, a space (usually in universities) where knowledge is shared without undue restriction-have not been adequately evaluated $^{11-13}$.

In the present analyses, we 'unpack' and contrast the motivations at play that are driving the pursuit for theragnostic patents and its bioethical corollaries in: (1) fundamental upstream basic research oriented to the discovery of genes for human diseases; and (2) downstream clinical applications at point-of-care as theragnostic tests to stratify patient populations for individualization of pharmacotherapy. We emphasize the need for integration, as well as the risk for excessive compartmentalization, of various biomarker technologies, and evaluate the subtle distinctions between DNA- and protein-based theragnostic tests.

\section{Upstream drug discovery research and theragnostic patents}

The search for genetic determinants of common complex human diseases reflects one of the pivotal upstream applications of pharmacogenomics. As DNA samples are being increasingly archived in pharmaceutical clinical trials, a sizable proportion of research resources are devoted to identifying the genes causally related to human diseases ${ }^{4}$. For many rare congenital monogenic human diseases (e.g., Duchenne muscular dystrophy), pre- or postnatal cytogenetic analysis of chromosomes or conventional clinical chemistry tests have existed for several decades. More recently, highthroughput genomic technologies spun off from the Human Genome Project (HGP) and the decreasing cost of genotyping have made possible the adoption of molecular genetic tests that can pinpoint the precise etiology or predisposition for a few adult-onset human diseases such as Huntington disease, certain familial forms of breast cancer, and Alzheimer disease $\mathrm{e}^{14}$. While genetic tests hold the promise of a more rational clinical forecast and management of disease risk in the future, they are also raising concerns about the provision of public healthcare services (reduced access) and the impact of market forces on the products of research (commercialization of technologies) and academic freedom. Some of these 


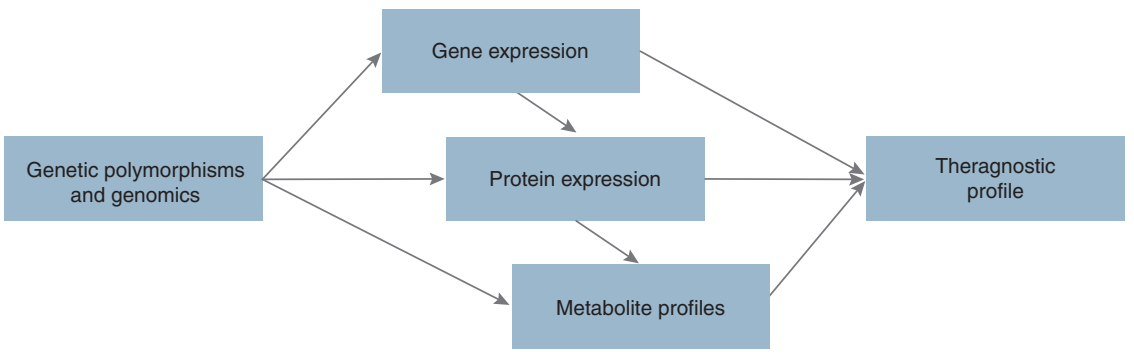

Static

Biomarker gradient

Figure 1 Hierarchy of biomarkers and their integration into theragnostic tests, from gene sequence (upstream or static marker) to downstream (dynamic) markers on gene and protein expression or cellular metabolites. A theragnostic profile is depicted as a synthesis of various biomarker tests that characterize an individual patient and her/his drug treatment outcome. The theragnostic profile may be heuristic in nature when only a singular biomarker is associated with treatment outcomes while more mechanistic insights can be achieved when biomarkers from different levels of the biological hierarchy corroborate and complement each other.

concerns have crystallized around the issue of commercial genetic testing for disease risk, as exemplified by the case of testing for hereditary breast cancer (BRCA testing) ${ }^{15}$.

In the mid-1990s, two genes (BRCA1 \& $B R C A 2)$ that greatly increase the risk for hereditary breast cancer were identified and sequenced. The BRCA genes were given broad patent protection in the US (and subsequently internationally in Canada, Europe, Australia, etc.), and granted to the biopharmaceutical company Myriad Genetics (Salt Lake City, UT), which had been involved in much of the initial research. This strong intellectual property protection has allowed Myriad to effectively control the BRCA testing market in the US; the commercial BRACAnalysis test is available only from Myriad or their licensees. Most recently, Myriad has licensed their test to San Franciscobased DNADirect, in order to provide services direct to consumers ${ }^{16}$.

Basic research into the function of the $B R C A$ genes or resulting proteins would be permissible without infringing on Myriad's patent rights, although there is still contention about what precisely constitutes basic research exclusions ${ }^{17}$. Myriad has, for example, signed agreements with the US National Institutes of Health and National Cancer Institute to provide sequencing at cost (US $\$ 1,200$ ) for research purposes ${ }^{18}$. However, research that results in a commercial or clinical service, defined by Myriad as any research in which a fee is charged for testing or in which results are provided to patients, would infringe on their patents. Notably, technology assessment research by third parties, for example to evaluate test performance metrics such as sensitivity, specificity, or positive predictive value, is particularly jeopardized. Moreover, research aimed at comparing the BRACAnalysis test against other testing methodologies would prove difficult, as the clinical nature of such a trial would constitute an infringement on Myriad's patents. Hence, the BRCA patents give Myriad the ability to constrain research-oriented applications of BRCA patents and particularly head-to-head comparisons of which genotyping methodology or test product is most informative for clinical management of the susceptibility to breast cancer.

In part due to Myriad's broad patent rights and the attendant concerns to ensure patients' access to affordable genetic testing for breast cancer risk, the European BRCA patents have been constrained or not enforced in recent years $^{15,19}$. In some sense the Myriad case may be thought of as an extreme scenario, and one from which industry and governments have learned. One specific consequence is that the initial enthusiasm for granting such broadly defined upstream patents on any and all forms of biological material has waned due to concerns for public good and scientific progress. More generally, there is a growing awareness that patents on genes and other biological materials can have an unfavorable effect on downstream genetics research and knowledge commons ${ }^{13,20}$. Thus, without an adequate conceptual framework on patents relating to theragnostic technologies, upstream patents on potential drug target genes or genetic methodologies for molecular definitions of human diseases (as in familial breast cancer) may lead to a monopoly on theragnostic tests. This may also dissuade some research laboratories from investigating otherwise potentially promising lines of inquiry for technology transfer towards downstream theragnostic products in the clinic $^{20}$. The Myriad patent case remains relevant since, in part, the current trends at the US Patent and Trademark Office (USPTO), and in patent offices in other countries, to grant more narrowly defined patents on biological materials were driven by this case ${ }^{21}$. According to some commentators, "No other event has had as big an impact on the human gene patent debate....and the case [Myriad] has thus become a 'harbinger' of the policy challenges created by gene patents" 21 .

More recent examples also support the idea that upstream theragnostic patents can limit translational applied clinical research. For instance, the SARS-associated coronavirus genome patents were filed by the US Centers for Disease Control (CDC) and the British Columbia Cancer Agency (BCCA) $)^{22-24}$, officially to ensure continued public access to the viral genome and pre-empt other entities from exerting restrictive or controlling rights. Notably, it is suggested that this type of patent is symptomatic of what is wrong with the [gene patent] system, when pre-emptive patents have to be filed to protect science and the public good ${ }^{22,23}$. A more worrying example is illustrated by the Australian company Genetic Technologies' patents on the non-coding regions of the human genome. Formerly conceived to be 'junk DNA', the biological role of non-genic segments of the genome is receiving increasing attention, and their patenting may significantly constrain the free design of primers for PCR analysis of coding regions of the genome $^{25,26}$.

A common thread in these two recent examples, however, is that broad theragnostic patents, particularly those granted on DNA and other biological materials, may serve as tollbooths that impede downstream research or discourage competition and innovation due to the broad exclusive rights granted to an individual scientist, institution or company (e.g., as in the case of Myriad). This 'anticommons' effect of upstream theragnostic patents is now increasingly being recognized. Patents with a broad scope may actually enclose the 'knowledge-commons' and inhibit technology transfer and development at a societal or macro level (i.e., as a contrast from the viewpoint of an individual investigator), such that the promises of new diagnostics and therapeutics are not realized $^{6,11,13}$.

\section{Theragnostic patents in downstream biomarker research to individualize drug therapy}

Pharmacogenomic-guided drug development represents a fundamental conceptual departure from conventional 'one-size-fits-all' clinical tri- 
als, as it aims to identify the subpopulations in whom drugs may display greater efficacy with lesser toxicity ${ }^{27}$. This is a favorable advance for rational therapeutics and optimal patient care but it also engenders varying degrees of trepidation among pharmaceutical companies and financial investors about proactive implementation in the clinic: pharmacogenomics may inevitably result in smaller economic markets for drugs introduced with an attendant genetic test predictive of drug efficacy or toxicity ${ }^{4,6,9}$. The pharma companies fervently respond that only drugs with large-scale markets allow recovery of the R\&D costs for new medications ${ }^{8,9}$, which can range from $\$ 400-800$ million according to different estimates ${ }^{28}$. While R\&D costs per se are not necessarily prohibitive to pursue targeted therapies, the pharmaceutical industry will still need to find mechanisms to maintain their growth rates in such niche markets defined by theragnostic tests. The ways in which upstream and downstream theragnostic patents are sought may play a decisive role in the development of focused therapeutic interventions in smaller markets that can benefit public good and industry growth equally.

As a contrast to arguments of market fragmentation, pharmacogenomics and related theragnostic technologies may enhance therapeutic differentiation and market penetration of new medicines ${ }^{29-31}$. Many of the currently marketed drugs, however, fall under the 'metoo' designation with comparable efficacy and safety profiles differing only in terms of slight changes in their chemical structures or pharmacophore composition ${ }^{32}$. Hence, in diseases or therapeutic areas characterized by me-too drugs, the diagnostic companies without a pharmaceutical pipeline may be more inclined to develop theragnostic tests that can impact more than one drug by virtue of being in the same therapeutic or chemical class. Conversely, in the case of large drug manufacturers, a theragnostic test for a me-too drug may be equally predictive of treatment outcomes for most if not all drugs within the same me-too category, redistributing the financial gains on the theragnostic test from an individual pharma company holding the theragnostic patent to multiple firms who manufacture similar me-too drugs. Consider, for example, a patient with major depression receiving the result of a theragnostic test on the serotonin transporter gene in relation to antidepressant response to paroxetine, a selective serotonin reuptake inhibitor (SSRI). In this case, the patient has the freedom afterwards to choose from among a host of comparable SSRI drugs without necessarily having to commit to paroxetine co-developed with the hypothetical theragnostic test. Therefore, the pursuit of theragnostic patents can also be shaped by the type of industry setting (e.g., diagnostic sector versus large pharma) as well as the type of pharmaceutical (e.g., me-too drugs) associated with theragnostic tests.

Regardless of the 'true' cost of drug development or the varied perceptions of the impact of pharmacogenomics or theragnostic tests on the economic promise of pharmaceuticals, the fact is that the blockbuster model of drug development with large-scale markets is increasingly less viable ${ }^{8,33}$. When new technologies such as pharmacogenomics and theragnostics enter the market, they can become 'paradigm-disruptive' forces that significantly undermine the traditional broadly defined market model of drug development and commercialization. Diverse and divergent diagnostic tests, multiple actors (e.g., biotech diagnostic companies, small and large pharma companies) seeking to create and protect their intellectual property, and changing social and political contexts (global demands for patent reform and licensing of drugs in the developing world) create an unstable environment for drug manufacturers. Despite the often very public proclamations about an interest in integrating pharmacogenomic research into drug development strategies, prospective stratification of patients using genetic tests in advanced stages of drug development with a view to proactive incorporation of pharmacogenomic data into drug labels is still rare ${ }^{33,34}$. This illustrates that there is a great uncertainty about how pharmacogenomic and theragnostic tests ought to be developed as functional commercial products.

It is noteworthy that multiplicity of diagnostic patents anticipated by the introduction of theragnostic technologies may also result in an 'anticommons effect' since most pharmacotherapeutic outcomes are polygenic or multifactorial in nature. If each segment of this expanding sphere of patentable biological elements along the biological dogma is held by different individuals, academic researchers or commercial firms, scientific advances in theragnostics can again be stifled, as in the case of broadly defined upstream gene patents. This is further supported by at least two seemingly divergent but complementary lines of evidence. First, the USPTO and patent offices in other countries increasingly favor narrowly defined gene patents, in part as a response to the Myriad case. Secondly, theragnostics is now introducing the need to characterize (and motivations to patent) downstream gene products such as mRNA, proteins or cellular metabolites to individualize drug therapy. Because time-dependent changes in gene expression or encoded proteins cannot always be accurately inferred from the upstream gene sequence, it is conceivable that there will be many more narrowly defined patents granted in the near future along the biological dogma from gene sequence to proteins and metabolites. Coupled with trends in patent offices in favor of narrowly defined gene patents, there will likely be a fragmentation of the diagnostic sector, as in the case of the blockbuster drugs and the niche therapies guided by theragnostic tests.

\section{Spectrum of theragnostic tests: Patenting across the biological dogma}

Many of today's most common diseases (including most forms of cancer, heart disease and psychiatric disorders) are known to arise not exclusively from either genes or environmental factors, but through a combination of the two (along with a significant amount of incalculable stochastic factors). Moreover, certain environmental exposures may only evoke illness when experienced during a critical period and in concert with a high-risk genetic background. Therefore, theragnostic tests predicated on genetic information alone would have a low a priori likelihood of capturing all of the predictable variance in a particular response outcome. Ideally, genetic tests could be fashioned to capture the entire heritable portion of a response variable (distributed across one or many genetic polymorphisms). In this context, genetic polymorphisms impart a constant or 'static' state of responsiveness that can be assayed once in each individual and presumed not to change over the course of the lifespan (barring de novo somatic mutations). The residual non-heritable portion of a given response phenotype must then be assayed by other means. To the extent that environmental (i.e., non-heritable) exposure influences response phenotypes by impinging on biological systems, this additional proportion of variance can be assayed through more 'dynamic' biomarker platforms, such as transcriptomics, proteomics, and metabolomics, each of which may be influenced by both genetic and environmental factors (Fig. 1). Thus, through a combination of static genetic and other dynamic biological '-omic' technologies (i.e., theragnostics), the potential to identify a more comprehensive set of predictors is maximized.

There are certain unique aspects of pharmacogenomic (and theragnostic) tests that differ from genetic testing for disease susceptibility. For all the parallels between genetic tests for disease risk and drug response, the latter are applied in reference to a drug that will be administered to patients in the immediate foreseeable future, while genetic testing for disease susceptibility usually predicts a risk in the distant future, often several years or decades away. This 'temporal dissociation' between the genetic test and the future disease occurrence 
may permit the estimation of the attendant cumulative disease risk with use of genetic data only; there is also a functional disconnect because the disease susceptibility test provides information which is rarely accompanied by effective treatment options.

In contrast to genetic testing for disease risk, pharmacogenomic tests are envisioned as being both temporally and functionally proximal. The purpose of a pharmacogenomic test is not to provide risk information as such, but to aid in the individualized prescription of a particular drug. Further, most drug effects are elicited within a matter of minutes, hours or days which may require a more precise estimation of the present or acute state of the pathophysiological pathway whose function is inferred through a genetic test. Hence, because the only barrier between the patient and drug safety or efficacy may be reliance on the accuracy of a pharmacogenomic test, clinicians need to know both the genetic variants in patients' DNA as well as the corresponding proteins encoded by the same genes. This is essential because (1) proteins are responsible for the eventual functional or clinical significance of genes and, (2) there may be marked differences or fluctuations in protein function (than what is predicted solely by gene structure) due to environmental factors or physiological feedback mechanisms that may influence posttranscriptional/posttranslational modification of gene products and proteins.

Further, an accurate prediction of drug effects may require a two-step complementary strategy involving, for example, both genetic and proteomic tests for the same gene and its protein product. This may create unprecedented challenges for patents and their legal defense. For instance, what are the implications of a biotechnology company attempting to develop a metabolomics-based, non-genetic 'dynamic phenotyping biomarker' for a gene patented hitherto for a static genotyping test to predict drug response or toxicity? There are presently no definitive answers to such emerging novel intellectual property issues associated with theragnostic tests.

\section{Conclusions and future perspectives}

Since its first appearance in the research literature in September 1997 (refs. 2,35), the term 'pharmacogenomics' has been hailed as a revolutionary enabling technology that can deliver highly customized drug therapies in the short term. Now, nearly a decade after its introduction, the more realistic expectation is that pharmacogenomics will complement efforts for rational individualization of drug therapy in conjunction with existing therapeutic monitoring tools and other novel biotech- nologies (e.g., proteomics and metabolomics). There is increasing support for the view that the human genome is highly dynamic and that gene expression, as well as the regulation of gene function, is subject to poorly understood plasticity. To achieve the much hoped for provision of personalized medicines, the role of environmental and social factors on both drug response and the human genome (and its expressed products, mRNA and proteins) requires detailed consideration. There is also growing recognition that the search for genetic biomarkers of outcomes associated with therapeutic interventions may carry the risk for compartmentalization among biomarkers through excessive reliance on a singular biotechnology. It is against this background that theragnostics is slowly emerging as a new concept to synthesize information from various biotechnologies directed at different levels of the biological dogma ranging from DNA (genomics), mRNA (transcriptomics), proteins (proteomics) or cellular metabolites (metabolomics).

Unlike mainstream genetic tests for disease susceptibility, the commercialization of theragnostic tests is inextricably linked with the deployment of patented pharmaceuticals. We suggest that theragnostic patents, particularly in the case of downstream applications at point-of-care, can be at variance with the traditional blockbuster model of drug development which stipulates the development of drugs for the entire population even though this approach yields modest therapeutic response and suboptimal drug safety ${ }^{8,10,33}$. Hence, a very different and unprecedented story is evolving for theragnostic patents at point-of-care: the traditional tight 'coupling' between patents and their subsequent commercialization may not always occur in clinical trials designed for the registration of new therapeutic candidates under the blockbuster model ${ }^{6}$. Experts in biotechnology patent law have thus slowly begun to point to this potential 'uncoupling' between the discovery of biomarkers on treatment outcomes and the necessary technology transfer to develop theragnostic products in the clinic. Instead, the downstream theragnostic patents on biomarker discoveries may remain primarily as in-house discoveries within the pharmaceutical industry to benefit future drug discovery efforts but without the accompanying translational clinical research for their development as a diagnostic kit for prediction of treatment response, failure or drug toxicity ${ }^{6,9}$. This uncoupling of biomarker discovery and necessary technology transfer towards their clinical application poses a threat to theragnostic product development. It is thus con- ceivable that academic research initiatives for biomarker discovery that consider both drug efficacy and broader functional treatment outcomes $^{29,36}$ will play a critical role in the development of theragnostic-guided personalized medicine.

In contrast to downstream patents on biomarkers associated with drug efficacy and safety, upstream patents in drug discovery and the identification of novel drug targets may be particularly welcomed by the pharmaceutical industry. This raises concerns over such patents becoming tollbooths that can increase costs for theragnostic tests in the clinic and slow or block downstream applied research. These nuanced contextual differences in applications of theragnostic patents in upstream research or at point-of-patient-care can shape the motivations at play, the strategies behind the patenting of genes, and the subsequent commercialization into theragnostic tests that may (or may not) become available to patients and consumers. The theoretical and practical framework on patents needs to incorporate implications of theragnostics on both upstream and downstream biomarker research while ensuring technology transfer by more than one stakeholder, to prevent future market monopoly and excessive premium pricing of theragnostic tests ${ }^{15}$.

As the pharmaceutical industry transitions from the blockbuster model towards targeted therapies with market shares that resemble orphan drugs, there is a parallel need to offer incentives to stakeholders who pursue theragnostic-guided drug development. The discipline of science and technology studies (STS) is already focused on the complex issues at the intersection of emerging biotechnologies, genetic research, bioethics, market forces and the pharmaceutical industry ${ }^{2,7,37}$. Unfortunately, the expertise in the STS research community does not always find its way into the mainstream medical research literature ${ }^{38,39}$. Such collaboration among geneticists, ethicists, applied pharmacologists and social scientists is essential for the equitable implementation of commercial theragnostic testing in the clinic, but also to prevent the risk of bioethics being used as a 'rubber stamp' that can 'deal with the issues' prior to the development of anticipated theragnostic-guided customized therapies. As witnessed during the initial planning and implementation stages of the HGP, we suggest that adequate attention and research resources should be made available to resolve these and similar policy and patent issues associated with theragnostic testing at the point-of-care. Additionally, these efforts should parallel the development of much needed prospective clinical investigations, designed primarily for 
the purpose of biomarker discovery, which can importantly contribute to development of targeted therapeutic interventions in the near future. We thus believe there is reason for guarded optimism that theragnostics may allow the synthesis of different types of biomarker data, DNA, protein or metabolomicbased, to achieve individualized therapeutics in medicine.

\section{ACKNOWLEDGMENTS}

All authors contributed to the ideas, critique and synthesis of the data discussed in the present review, as well as the specific considerations involving the role of gene patents on theragnostic tests and nuanced distinctions among different types of biomarkers. The concepts and ideas presented in the present analyses were developed and contextualized during the 2005 Canadian Bioethics Society Annual Meeting in Halifax, Nova Scotia (V.O. and B.W.-J.), a research sabbatical (C.R.) and interdisciplinary collaborative efforts led by the Working Group on Personalized Medicine in Major Psychosis (S.J.G., J.B.L. and M.T.T.) at the Center for Behavioral Genomics, Department of Psychiatry, UCSD.

Supported in part by research grants from the VISN 22 Mental Illness Research, Education, and Clinical 윽 Center (V.O.), the Canadian Institutes of Health Research and the Faculty of Medicine, Université de Montréal (B.W.-J.) and a Merit Review operating grant from the Veterans Affairs Office of Research and Development (C.R.). The funding sources had no role in the analyses, synthesis or critique of the data.
1. Brown, N. Science Studies 16, 3-21 (2003).

2. Hedgecoe, A.M. Science Technol. Human Values 28, 513-537 (2003).

3. Hedgecoe, A. \& Martin, P. Soc. Stud. Sci. 33, 327364 (2003).

4. Williams-Jones, B. \& Corrigan, O.P. Am. J. Pharmacogenomics 3, 375-383 (2003).

5. Nightingale, P. \& Martin, P. Trends Biotechnol. 22, 564-569 (2004).

6. Eisenberg, R.S. Pharmacogenomics 3, 571-574 (2002).

7. Hedgecoe, A. The Politics of Personalised MedicinePharmacogenetics in the Clinic. Cambridge Studies in Society and the Life Sciences, 1-208 (Cambridge University Press, Cambridge, UK; 2004).

8. Service, R.F. Science 303, 1796-1799 (2004).

9. Sherrid, P. US News World Report 131, 30-32 (2001).

10. Ozdemir, V. et al. Curr. Pharmacogenomics 3, 53-71 (2005).

11. Williams-Jones, B. J. Med. Ethics 31, 249-250 (2005).

12. Ozdemir, V. et al. Expert Opin. Pharmacother. 7, 119-133 (2006).

13. Williams-Jones, B. \& Ozdemir, V. in Ethics and Law of Intellectual Property: Current Problems in Politics, Science and Technology. (eds. Lenk, C., Hoppe, N. \& Andorno, R.) 172-209 (Ashgate, London; 2006).

14. Evans, J.P., Skrzynia, C. \& Burke, W. Br. Med. J. 322, 1052-1056 (2001).

15. Williams-Jones, B. \& Burgess, M.M. Kennedy Inst. Ethics J. 14, 115-142 (2004).

16. Associated Press. DNA testing goes DIY. Wired News, March 7, 2005. Available at: http://www.wired.com/ news/medtech/0,1286,66822,00.html (accessed 28 April, 2006).

17. Baird, P.A. Perspectives in Biology and Medicine 41, 391-408 (1998).

18. Reynolds, T. J. Natl. Cancer Inst. 92, 596 (2000).
19. Williams-Jones, B. \& Graham, J.E. New Genet. Soc. 22, 271-296 (2003).

20. Merz, J.F. et al. Nature 415, 577-579 (2003).

21. Caulfield, T. Community Genet. 8, 223-227 (2005).

22. Gold, E.R. Lancet 361, 2002-2003 (2003).

23. World Health Organization resource on patentability of the SARS virus genome. Available at: http://www. who.int/ethics/topics/sars_patents/en/ (accessed 28 April, 2006).

24. Anon. Nature 423, 207 (2003).

25. Dennis, C. Nature 423, 105 (2003).

26. Paradise, J. \& Janson, C. Nat. Rev. Genet. 7, 148154 (2006).

27. Ozdemir, V., Shear, N.H. \& Kalow, W. Drug Safety 24 75-85 (2001).

28. DiMasi, J.A., Hansen, R.W. \& Grabowski, H.G. J. Health Econ. 22, 151-185 (2003).

29. Albers, L.J. \& Ozdemir, V. Curr. Med. Chem. 11, 297-312 (2004).

30. Evans, W.E. \& McLeod, H.L. N. Engl. J. Med. 348 538-549 (2003).

31. Goldstein, D.B., Tate, S.K. \& Sisodiya, S.M. Nat. Rev Genet. 4, 937-947 (2003).

32. Angell, M. CMAJ 171, 1451-1453 (2004)

33. Ozdemir, V. \& Lerer, B. in Pharmacogenomics, Edn. 2. (eds. Kalow, W., Meyer, U.A. \& Tyndale, R.F.) 13-50 (Francis and Taylor, New York; 2005).

34. Sanders, R. Pharmacogenomics 4, 541-545 (2003).

35. Marshall, A. Nat. Biotechnol. 15, 829-830 (1997).

36. Lebowitz, B.D. \& Rudorfer, M.V. J. Clin. Psychopharmacol. 18, 1 (1998).

37. Smart, A. et al. Bioethics 18, 322-342 (2004).

38. Latour, B. in We have never been modern. (Harvard University Press, Cambridge, MA; 1993).

39. Latour, B. in Science in Action: How to Follow Scientists and Engineers through Society. (Harvard University Press, Cambridge, MA; 1987). 